\title{
The Urgency of Public Transportation in Terms of Supervision in Accordance with Health Protocols
}

\author{
Endriy Artem ${ }^{1}$, Voktir Busyedin ${ }^{1}$ \\ ${ }^{1}$ Faculty of Pediatrics and Public Health, Donetsk National Medical University, Ukraine
}

\begin{abstract}
The goal of this study was to ascertain the critical nature of public transportation under the supervision of a health protocol. During the COVID-19 pandemic's New Normal phase, the government issued an appeal addressing health procedures for individuals who are active in public spaces or who use public facilities. This protocol also applies to the public transportation industry, specifically the bus industry. Health rules for bus use must be strictly adhered to and monitored in order to prevent the virus from spreading. The health protocol is implemented to offer a sense of security for public bus riders without jeopardizing the quality of service. A series of laws limiting capacity and enforcing strict adherence to health protocols for all modes of public transportation.
\end{abstract}

Keywords: Public Transport, Health Protocol, Society

Received: September 12, 2021

Revised: September 27, 2021

Accepted: October 9, 2021

\section{Introduction}

Today's transportation modes are an example of technology advancement that makes it easier for humans to perform their daily activities. Several kinds of transportation that exist today, such as airplanes, ships, motorcycles, and automobiles, have undergone numerous modifications in terms of technology, security, and comfort, ensuring that users of these modes of transportation feel safe and secure. The population's mobility from rural to urban regions or between cities, which is developing rapidly, is in desperate need of this mode of transportation to help meet basic needs (Luo et al., 2018). It is indisputable that the majority of citizens rely on land transportation to assist them in completing the majority of public activities such as job, education, or vacation. The most often utilized mode of public transportation in this instance is the public bus, which transports passengers to and from destinations within and beyond the city. Buses as public transit is one strategy for reducing congestion in these two megacities. This is unquestionably supported by both the government and citizens of each city, provided that the level of security and services supplied are adequate. However, many businesses providing these transportation services continue to deliver substandard and unsafe services, prompting many individuals to choose for alternative modes of transit or private vehicles. During the COVID-19 pandemic's New Normal phase, the government issued an appeal addressing health standards for persons who work in public areas or who use public facilities, where customer satisfaction is one factor that contributes to a company's positive image.

People are needed to adapt to a new way of life during this epidemic in order to make peace with Covid-19. What is intended by the term New Normal is an activity or behavior taken by the Community Empowerment community and any associated parties in the region in order to carry out new and different daily patterns, work patterns, or new lifestyles. If this is not done, the chance of transmission increases. The New Normal's objective is to keep people productive and safe from Covid-19 during the epidemic. Additionally, in order to make the New Normal more accessible to the public, a suitable phrase for it was sought, which is Adaptation of New 
Habits. The goal of developing new habits is to enable us to work, learn, and engage in productive activities during the Covid-19 epidemic era. Covid-19 has caused a variety of illnesses affecting the respiratory, gastrointestinal, hepatic, and neurological systems in humans and animals with varied degrees of severity. The existence of a work-from-home policy designed to avert a Covid-19 epidemic has a detrimental effect on a variety of public transit services. The reason for this is that since the policy's inception, the number of passengers on public transit has declined significantly (Dai et al., 2021). The subsequent effect has been a major fall in the salary of public transportation drivers (Sadowsky \& Nelson, 2017). Community service initiative in the form of health protocol education in public transportation, with the primary focus on public transportation drivers (Levasseur et al., 2017; Iclodean eta 2020). Counseling as a kind of education or public education, followed by participatory actions, has the potential to increase understanding and awareness of the necessity of clean and healthy living behaviors, particularly in the context of the Covid-19 pandemic.

A collection of policies limiting capacity and strictly enforcing health protocols for all modes of public transportation. This is what inspires us to raise public knowledge and understanding of the numerous benefits of mass public transportation. Capacity restrictions and enforcement of health rules during the Covid-19 pandemic are part of an effort to maintain public faith in mass public transportation in order to prevent the Covid-19 virus from being transmitted via mass public transportation (Tirachini \& Cats, 2020).

\section{COVID-19 and Public Transportation}

When it comes to the pathogen's capacity to travel, buses and trains are unquestionably the finest modes of transmission - a January investigation of infection in nine passengers on a longdistance bus in Hunan became famous in this regard; the research has been temporarily halted without explanation.

Public transportation has been banned in a number of Asian cities, including Wuhan, Huanggang, and Delhi, to contain the infection. While a complete closure of public transportation is not a solution that all cities afflicted by the pandemic will take, it is critical to identify methodical areas of action to minimize dangers to public transportation employees and passengers. To ensure an organized reaction and effective implementation of measures by public transportation firms and authorities, a viral or infectious pandemic response plan should serve as the foundation for action and implementation (Nguyen et al., 2021). Additionally, any steps taken by government organizations and public transportation firms to safeguard the safety of employees and passengers and to combat the spread of COVID-19 must be based on a thorough impact assessment. Actions must take into account their social, environmental, climate, and economic consequences.

\section{Staff, Infrastructure and Passenger Protection Needs}

In public transportation, the most valuable asset is the workforce. As a result, they must be protected specifically, both as individuals and in their capacities as drivers, supervisors, and managers, among others. It is inherent in the nature of public transportation staff to be in intimate contact with their customers, namely passengers. As a result, protective measures cannot account for all possible dangers.

International organizations such as the American Public Transportation Association's Transportation Research Agency and the International Organization for Public Transportation Authorities and Operators publish fact sheets and other information and keep an eye on the situation. It is critical to conduct regular searches on their site for information. 
Restriction measures implemented to contain the spread and impact of Covid-19 have had a profound effect on people's life and the way energy is used across the economy. One of the most significant consequences has been a fall in passenger transport demand, owing to a mix of government lockdowns and fears of getting and spreading the virus while using mass transportation options. While freight transport has also decreased in recent years, the drivers of freight activity during this crisis are multifaceted, influenced by supply and demand side issues, as well as the necessity to maintain key services. In comparison, passenger transportation is frequently voluntary and is more influenced by individuals' decision-making processes. As a result, this study will concentrate on passenger transportation.

The issue has impacted every mode of transportation, from private automobiles and public transportation in cities to buses, trains, and airplanes on a national and worldwide scale. At the end of March 2020, global road transport activity was nearly 50\% below the 2019 average, while commercial aviation activity was nearly $75 \%$ below the 2019 normal in mid-April 2020 . Additionally, public transportation was impacted. For example, the UK's rigorous lockdown in March 2020 resulted in a 95 percent decline in underground travel in the capital. This is corroborated by data1 from a popular transportation planning smartphone app, which indicates that travel has decreased by more than $90 \%$ in many of the world's largest cities since the crisis began.

\section{The urgency of public transportation in the midst of a pandemic}

Transport in Asia and the Pacific, which details the pandemic's tremendous influence on transportation, including how fast lockdowns caused millions of people to work from home overnight this year, schools shifted to online learning, and consumers flocked to online shopping and food delivery. While public transportation was once considered the most ecologically friendly, efficient, and economical mode of transportation, early patterns in reopened cities indicate that public transportation remains relatively risky and is not recovering as soon as it could. private vehicles, cycling, and walking are all acceptable modes of transportation.

The two primary difficulties ahead are improving public transportation capacity in order to meet safe distance regulations and regaining public faith in public transportation (Abdallah, 2017). In the short term, additional efforts should be made to reassure public transportation customers of their safety and to demonstrate that public transportation is clean and safe. Longterm, advancements in technology, big data, artificial intelligence, digitalization, automation, renewable energy, and electric power have the potential to provide new solutions to address shifting demands, resulting in smarter cities.

While global economic collapse has been exacerbated by harsh lockdown measures, satellites have documented how $\mathrm{CO} 2$ and other air pollution have decreased dramatically, resulting in stunning blue skies above many cities. However, as cities reopen, traffic congestion increases. For example, as of early April 2020, Beijing's traffic levels had surpassed those of the same period in 2019. When seen broadly, this trend has the potential to undermine decades of efforts to promote sustainable development and more efficient modes of urban movement.

According to the report, cities have little opportunity to promote the adoption of low-carbon alternatives in order to maintain the improved air quality conditions achieved at the pandemic lockdown's peak. Public transportation may play a critical role in improving overall health and well-being by promoting more active, clean cars, providing quality alternatives to public transportation, and creating a better environment for non-motorized modes of transportation such as walking and cycling. 
The research stated that passengers' trust in public transportation must be reestablished by safety measures such as cleaning, thermal scanning, tracking, and facial coverings. Additional research to determine how protective and preventive measures might be scaled up to allow for the relaxation of safe distance standards will assist in mitigating capacity constraints. A likely future trend is the consolidation and rationalization of services in order to better fulfill emerging travel demand patterns and habits.

As countries reach the "recovery" phase, more safeguards and innovative technology must be deployed to enable contactless procedures and quick reaction. Demand management strategies can aid in the management of crowds in public transportation systems and airports. Additionally, non-motorised transport capacity can be developed to absorb excess demand from public transportation.

Because mass public transportation is critical to a big portion of the economy, government policies and financial support are critical during this period to ensuring that public transportation providers stay viable and continue to facilitate sustainable passenger and freight movement.

\section{The effectiveness of implementing health protocols on public transportation during the pandemic}

\section{Public transport planning takes social distancing into account}

Public transport planning on a strategic level refers to the process of designing a public transportation network, which includes identifying public transportation terminals and developing public transportation routes requires additional adjustments (Okraszewska et al., 2018; Saif et al., 2019).

Despite the necessity of adjusting the public transportation network, long-term decisions such as station locations or which roads/rails must be followed by bus/train lines cannot be easily modified to the pandemic's needs. Due to the constraints imposed by an already constructed network, public transportation service providers' strategic options are limited. However, as previously stated, some operators have totally discontinued or altered particular line services by selectively closing stations in response to passenger requests.

Although station decisions are decided depending on the transport operator's local expertise, there is a substantial body of literature on determining which stations to skip in order to increase service efficiency based on prevailing demand trends. Although the literature does not address the impacts of social separation, there are various papers that recommend which stations to bypass for specific demand patterns. Stop-skipping has been utilized consistently at the operational level to choose which stops to skip on a particular bus or train journey while taking into account the negative repercussions for individuals who use these stations. At the strategic offline level, public transportation service providers are willing to identify which stops should be fully eliminated in order to save money or mitigate transmission risk. To create a strategic schedule of skipped stops for all daily travels, the process for determining the strategy for missed stops considers a variety of important performance factors. These key performance indicators should include not only waiting time and time spent in a passenger vehicle but also vehicle travel time. They should also take into account issues of equity pertaining to vulnerable groups. Additionally, while making stop-jump decisions, critical employees should be considered. 


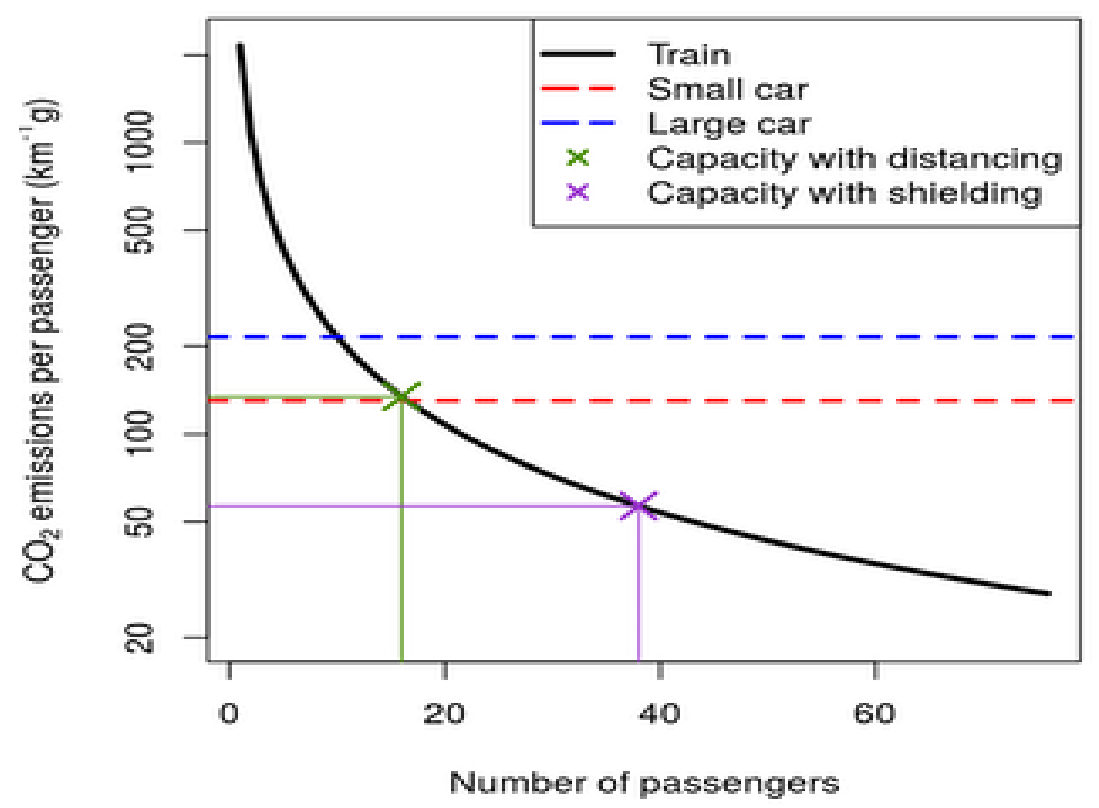

Figure 1. Emissions per passenger of a variety of modes of transport with a social distance of two metres

\section{Source: Henley, Moore and Ostler (The Conversation)}

\section{Tactical planning Service frequency}

At the tactical planning stage, service providers have a far higher degree of freedom. This includes a decrease in service frequency, modifications to vehicle timetables and schedules, and a decrease in the total duration of daily operations. All of these metrics are converted to dimensions of service capability. Several train and bus providers have cut their service frequency to less than a third of what it was pre-pandemic. However, such decisions are rarely made on the basis of a system-wide analysis, and public transportation service providers urgently require tools to assist them in making the most efficient use of available resources.

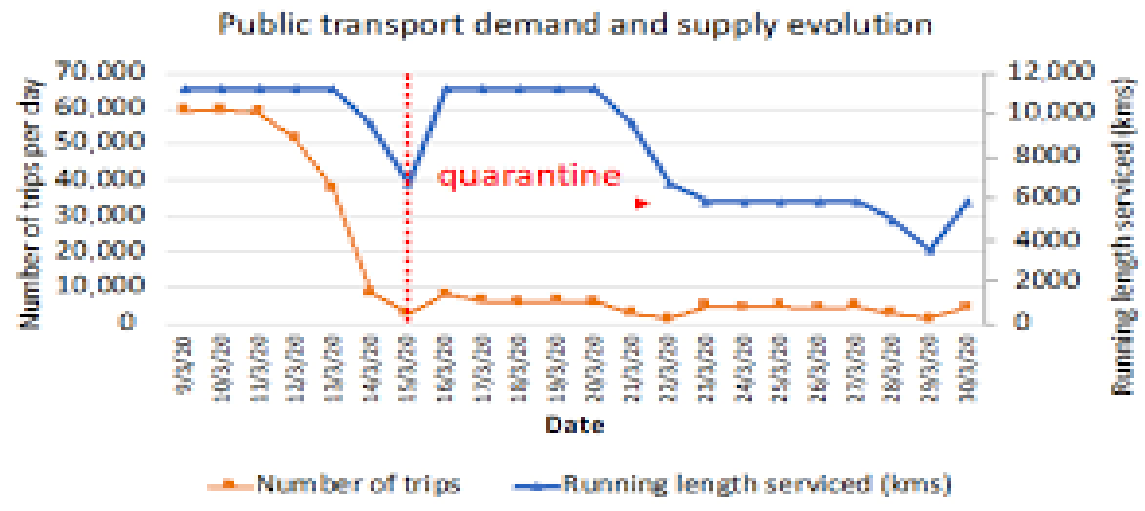

Figure 2.Evolution of public transport users

Source: www.moovit.com

Numerous models attempt to find the appropriate service frequency by balancing general passenger expenses e.g., waiting time against operational costs eg number of vehicles deployed and total km-trips. Previously published work concentrated on determining the frequency of a single line at a time. Given the problem's reduced complexity, offered closed-form statements 
that do not necessitate the solution of sophisticated mathematical programs for example maximum load and load profiling methods.

Several methods have been presented during the last decade for creating service frequency throughout a network by evaluating the optimal resource allocation for scarce resources (Gkiotsalitis \& Cats, 2021). provides a two-level programming technique for the frequency setting problem that minimizes total passenger journey time while adhering to the overall fleet size limitation. Additionally, it examines frequency management as a means of balancing passenger demand and available supply. We investigated optimal frequency management and mixed fleet allocation and provided a reliable frequency management model that takes service volatility into consideration. Finally, utilizing meta-heuristics, the problem of frequency setting is merged with route design.

The models described above define a set service frequency that is applicable to all line stations. Develop a frequency management model that incorporates flexible virtual lanes namely shortturn lanes and/or interlining lanes to efficiently and effectively distribute available vehicle/driver resources

\section{Conclusion}

Public transit is one kind of transportation that contributes to congestion reduction. However, there are still a number of firms that provide transportation services that do not provide highquality or safe services, forcing many people to rely on alternative modes of transportation or private vehicles. During the COVID-19 pandemic's New Normal phase, the government issued an appeal addressing health procedures for individuals who are active in public spaces or who use public facilities. The goal of developing new habits is to enable us to work, learn, and engage in productive activities during the Covid-19 pandemic. Work from home policies have a detrimental effect on a number of public transit services. Numerous offices and businesses continue to operate while adhering to health rules. However, in the midst of a pandemic, public health and safety take precedence. As a result, health regimen application on public transit becomes extremely rigorous. As a result, transportation will continue to operate while efforts are made to avoid covid-19.

\section{References}

Abdallah, T. (2017). Sustainable Mass Transit: Challenges and Opportunities in Urban Public Transportation. Elsevier.

Dai, J., Liu, Z., \& Li, R. (2021). Improving the subway attraction for the post-COVID-19 era: The role of fare-free public transport policy. Transport Policy, 103, 21-30.

Gkiotsalitis, K., \& Cats, O. (2021). Optimal frequency setting of metro services in the age of COVID-19 distancing measures. Transportmetrica A: Transport Science, 1-21.

Iclodean, C., Cordos, N., \& Varga, B. O. (2020). Autonomous shuttle bus for public transportation: A review. Energies, 13(11), 2917.

Levasseur, M., Dubois, M. F., Généreux, M., Menec, V., Raina, P., Roy, M., ... \& St-Pierre, C. (2017). Capturing how age-friendly communities foster positive health, social participation and health equity: a study protocol of key components and processes that promote population health in aging Canadians. BMC public health, 17(1), 1-11.

Luo, J., Zhang, X., Wu, Y., Shen, J., Shen, L., \& Xing, X. (2018). Urban land expansion and the floating population in China: For production or for living?. Cities, 74, 219-228. 
Nguyen, T. V., Dai Tran, Q., Phan, L. T., Vu, L. N., Truong, D. T. T., Truong, H. C., ... \& Pham, Q. D. (2021). In the interest of public safety: rapid response to the COVID-19 epidemic in Vietnam. BMJ global health, 6(1), e004100.

Okraszewska, R., Romanowska, A., Wołek, M., Oskarbski, J., Birr, K., \& Jamroz, K. (2018). Integration of a multilevel transport system model into sustainable urban mobility planning. Sustainability, 10(2), 479.

Sadowsky, N., \& Nelson, E. (2017). The impact of ride-hailing services on public transportation use: A discontinuity regression analysis.

Saif, M. A., Zefreh, M. M., \& Torok, A. (2019). Public transport accessibility: a literature review. Periodica Polytechnica Transportation Engineering, 47(1), 36-43.

Tirachini, A., \& Cats, O. (2020). COVID-19 and public transportation: Current assessment, prospects, and research needs. Journal of Public Transportation, 22(1), 1. 